\title{
The effects of coupling misalignment on vibration of rotor bearing system
}

\author{
Eui-Wook Jeong, Sun-Woong Kwon, Van-Canh Tong and Seong-Wook Hong"
}

\begin{abstract}
Misalignment is one of the most influential factors that affect the vibration of a rotor bearing system. This paper presented a combined analytical and experimental approach to investigate the effect of the coupling misalignment on the vibration behavior of a rotor supported by tapered roller bearings. First, a theoretical model was developed to predict the vibration in the rotor system subjected to angular misalignment in the flexible coupling. Then experimental measurements were made from an instrumented test-rig to verify the model. Finally, extended simulations were performed to investigate the effect of misaligned amplitude, rotational speed disk position, bearings distance, and arrangement on the vibration behavior of the rotor. Simulation results showed that the coupling misalignment leads to vibration with multiple harmonics of rotational speed, and that misaligned coupling introduced anisotropic nature in vertical and horizontal vibration responses of the rotor.
\end{abstract}

Keywords—misalignment, vibration, rotor system

\section{Introduction}

The occurrence of misalignment between a drive and a driven shaft often generates additional fluctuating forces and moments at coupling location [1]. Consequently, misalignment contributes to oscillating load and thus premature failure of components in rotating machines. Misalignment has a significant influence on the life, particularly on the bearings. Therefore, the problem associated with misalignment has attracted great attentions from the designers and engineers [2-5].

In this study, the effects of angular misalignment in a flexible coupling on the vibration of a rotor system were investigated. An analytical model is developed to estimate the vibration of the system with coupling misalignment. The model was verified by mean of experimental measurement. Then the effects of misalignment amplitude and rotor rotational frequency on the rotor vibration responses were discussed.

\section{Numerical model of rotor bearing system}

\section{A. System equations of motion}

This section presents the theoretical model for obtaining vibration responses of the rotor bearing system. Figure 1 shows the investigated rotor system which consists of a rigid disk, a shaft, and a pair of supporting TRBs in face-to-face arrangement. The rotor shaft is connected to the driving

Eui-Wook Jeong, Sun-Woong Kwon, Van-Canh Tong and Seong-Wook Hong Kumoh National Institute of Technology

Republic of Korea motor via a four pin flexible coupling. To make the system equations of motion, this study used the finite element method, which has been effectively applied for vibration analysis of rotor bearing systems [6,7]. The equations of motion for shaft elements, disk, and bearings are constructed and assembled to make the equations of motion for the whole rotor system as follows:

\section{Shaft element}

The shaft is discretized into a finite number of elements, in which each element is represented by two nodes. Then, the equation of motion for a finite shaft element is represented as

$\left[\begin{array}{cc}m^{s} & 0 \\ 0 & m^{s}\end{array}\right]\left\{\begin{array}{l}\ddot{y}^{s} \\ \ddot{z}^{s}\end{array}\right\}+\Omega\left[\begin{array}{cc}0 & g^{s} \\ -g^{s} & 0\end{array}\right]\left\{\begin{array}{l}\dot{y}^{s} \\ \dot{z}^{s}\end{array}\right\}+\left[\begin{array}{cc}k^{s} & 0 \\ 0 & k^{s}\end{array}\right]\left\{\begin{array}{l}y^{s} \\ z^{s}\end{array}\right\}=\left\{\begin{array}{l}f_{y}^{s} \\ f_{z}^{s}\end{array}\right\}$.

where $m^{s}$ and $g^{s}$ are the mass and gyroscopic matrices of shaft element, respectively. $\{y\}^{\mathrm{T}}$ and $\{z\}^{\mathrm{T}}$ represent the displacement vectors in $X-Y$ and $X-Z$ planes. The force vector on the right hand side of Eq. (1) is the external force on the shaft elements which may be unbalance force, coupling force, and other external forces on the shaft elements.

\section{Disk}

The disk is located at a node as shown in Fig. 1. The equation of motion for the rigid disk with constant rotational speed is given by

$$
\left[\begin{array}{cc}
m^{d} & 0 \\
0 & m^{d}
\end{array}\right]\left\{\begin{array}{l}
\ddot{y}^{d} \\
\ddot{z}^{d}
\end{array}\right\}+\left[\begin{array}{cc}
0 & g^{d} \\
-g^{d} & 0
\end{array}\right]\left\{\begin{array}{l}
\dot{y}^{d} \\
\dot{z}^{d}
\end{array}\right\}=\left\{\begin{array}{l}
f_{y}^{d} \\
f_{z}^{d}
\end{array}\right\} .
$$

where $m^{d}$ and $g^{d}$ are the mass and gyroscopic matrices of disk element, respectively.

\section{Bearing}

Similar to the disk, the bearing is located at a node. The equation of motion for the bearing is represented as

$$
\left[\begin{array}{cc}
c_{y y}^{b} & c_{y z}^{b} \\
c_{z y}^{b} & c_{z z}^{b}
\end{array}\right]\left\{\begin{array}{l}
\dot{y}^{b} \\
\dot{z}^{b}
\end{array}\right\}+\left[\begin{array}{cc}
k_{y y}^{b} & k_{y z}^{b} \\
k_{z y}^{b} & k_{z z}^{b}
\end{array}\right]\left\{\begin{array}{l}
y^{b} \\
z^{b}
\end{array}\right\}=\left\{\begin{array}{l}
f_{y}^{b} \\
f_{z}^{b}
\end{array}\right\}
$$

where $\left\{f_{y}^{b} f_{z}^{b}\right\}^{\mathrm{T}}$ is the bearing external force vector. The matrices $c_{i j}{ }^{b}$ and $k_{i j}{ }^{b},(i, j=y, z)$ represent the bearing damping and stiffness matrix, respectively. The bearing stiffness elements can be obtained from the bearing model [8, 9]. It is assumed that the bearing stiffness coefficients remained constant during the rotor operation.

\section{Equation of motion}

Combining the shaft element and bearing equations, the assembled equation of motion of the rotor bearing system is obtained as

$$
[M]\{\ddot{q}\}+\Omega[G]\{\dot{q}\}+[K]\{q\}=\{f\}
$$




\section{B. Coupling misalignment}

Misalignment in rotating machinery generates reactive forces on the shaft ends by the coupling. When the driving motor shaft and driven rotor shaft is misaligned by angle $\theta$, the static forces and moments acting on the rotor shaft end is calculated by [4].

$$
\begin{aligned}
& M_{x}=-K_{b} \theta, M_{y}=T_{q} \sin \theta \\
& F_{x}=M_{y} / Z, F_{y}=-M_{x} / Z
\end{aligned}
$$

where $K_{b}$ and $Z$ are the bending stiffness, and the center of articulation of the coupling, respectively. $T_{q}$ is the transmitted torque. When the shaft rotates, those forces become periodic with multiple harmonics of rotational speed. A method for determination of the frequency order of coupling forces and moments was described in Refs. [1-3]. For the coupling with angular misalignment, the time varying force with frequency order up to $7 \mathrm{X}$ components of the reaction forces has been observed [2, 3]. However, the amplitudes of force components beyond $4 \mathrm{X}$ is relatively small. Therefore, for numerical analysis, the coupling forces and moments comprised up of $1 \mathrm{X}$ to $4 \mathrm{X}$ components are considered. These are incorporated into the excitation force vector of the equation of motion.

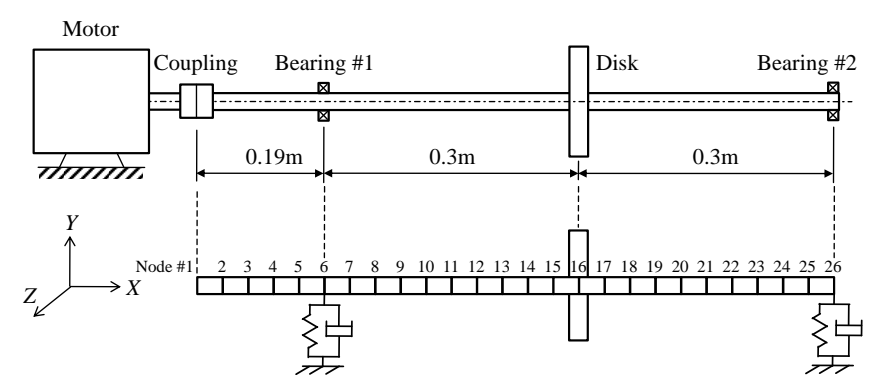

Figure 1. Rotor system modeling

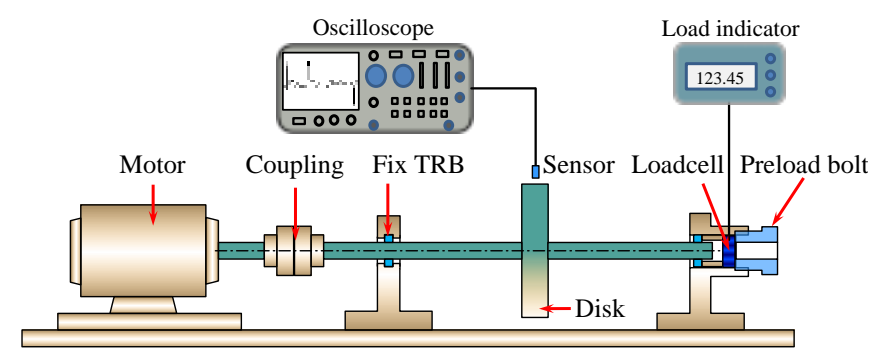

Figure 2. Experiment setup

\section{Experimental setup}

Figure 2 shows the experimental setup for measuring vibration responses of a rotor system. The position of bearing housings and motor base can be adjusted using screw bolts so as to change the misalignment condition of the system. Axial preload is applied through outer race of the right-hand side TRB by a preloading device using threaded preload screw bolt. The preload is measured by a compression type load-cell (LCWD-10K, Omega), which is located between the preload screw bolt and cylinder. A load indicator is used to monitor the preload force. Axial preload is transferred to the TRB via an annular cylinder. Vibration of the disk is measured using gap sensors. The output voltage of the sensor is collected using an oscilloscope.

\section{Results and discussion}

This section presents the numerical and experimental results of rotor vibration responses. First, the theoretical model presented in the previous section is verified using experimental measurements. Then extended simulations are performed to investigate the effects of coupling misalignment angle, rotational speed, disk position, bearings span distance and arrangement on the vibration responses of the rotor.

\section{A. Model verification}

The shaft of experimental system has a bow error of $e=$ $102.4 \mu \mathrm{m}$ estimated at the disk position or mid-span of the bearings. Figure 3 shows the test result of horizontal vibration of the disk when the coupling is well-aligned and the shaft is slowly rotating at $1.46 \mathrm{~Hz}$. At this speed, the dynamical effect on the disk vibration is assumed to be negligible. Therefore, vibration is caused only by the bow error in the shaft. When the shaft is rotating at a rotational frequency of $f$, the vibration caused by shaft error may be expressed by

$$
\left\{\begin{array}{l}
x_{e}=e \sin (\omega t) \\
y_{e}=e \cos (\omega t)
\end{array} .\right.
$$

This error must be added to the computational results of the disk vibration since the model does not include such error effect. In addition, the bow error in the shaft leads to additional unbalance load acting on the disk, which needs to be considered for the simulation model when comparing the numerical and experimental results. The unbalance force acting on the disk is written by

$$
\left\{\begin{array}{l}
F_{x}=m_{d} e \omega^{2} \sin (\omega t) \\
F_{y}=m_{d} e \omega^{2} \cos (\omega t)
\end{array} .\right.
$$

where $m^{d}$ is the mass of the disk and $\omega$ is the angular velocity of the rotor, $\omega=2 \pi f$.

Figure 4 shows the frequency spectrum of disk vibration with the coupling misalignment angle of $\theta=0.2 \mathrm{deg}$. The rotational frequency of the rotor shaft is $f=10 \mathrm{~Hz}$ and bearing preload is $2450 \mathrm{~N}$. The simulation and experiment results show an overall good agreement. In general, coupling misalignment introduces multiple harmonics of rotational frequency to the system vibration. The vibration component at the system natural frequency $f_{\mathrm{n}}=48.33 \mathrm{~Hz}$ seems not occur in the experimental results but it is clearly visible in the simulated spectrum. Figure 5 shows the simulated vibration spectrum of the disk after subtracting the bow error of the shaft. The vibration at the natural frequency is significant. The difference between the experiment and simulation may be due to the effect of bearing stiffness and damping. The present study assumed constant bearing stiffness and zero damping. However, the actual bearing stiffness coefficients may be time-varying due to varying bearing loading and displacements. In-depth investigation on the involvement of time varying bearing stiffness is subjected to future work. 


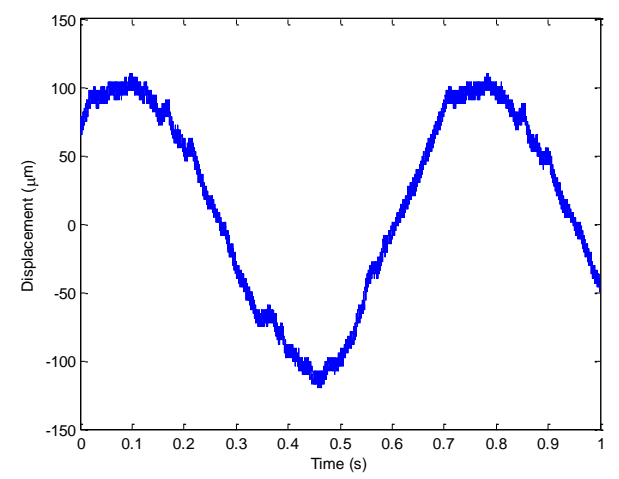

Figure 3. Disk displacement caused by shaft bow error
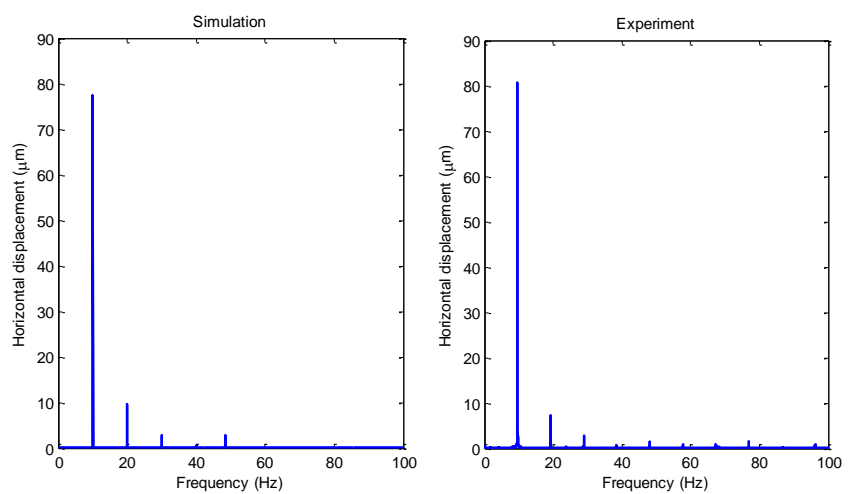

Figure 4. Frequency spectrum of horizontal vibration at the disk $(f=10 \mathrm{~Hz}$, $\theta=0.2 \mathrm{deg})$
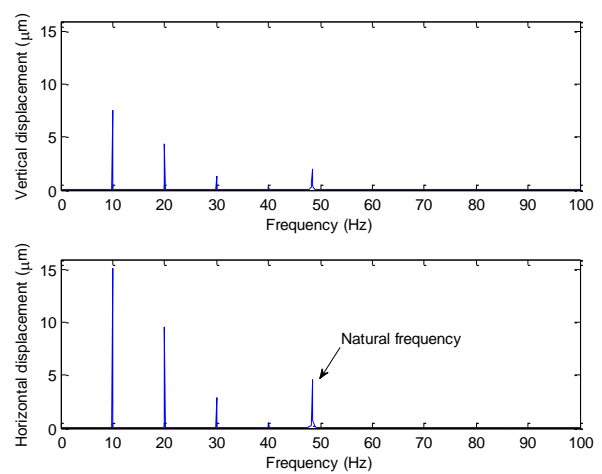

Figure 5. Frequency spectrum of disk vibration after subtracting the shaft straightness error $(f=10 \mathrm{~Hz}, \theta=0.2 \mathrm{deg})$

\section{B. Effect of the coupling misalignment angle on rotor vibration}

This section presents the effect of coupling misalignment on vibration of the rotor system. The rotational frequency of rotor and preload of the TRBs are kept constant at $10 \mathrm{~Hz}$ and $2450 \mathrm{~N}$, respectively. The simulations are performed for the rotor system without shaft bow error.

Figure 6 shows the frequency spectrum of disk vibrations calculated at the misalignment angle of coupling of $0.05,0.1$ and $0.15 \mathrm{deg}$. Increasing misalignment angle leads to the increase of vibration amplitudes of all frequency components. This is explained by the increase of coupling load acting on the shaft end.
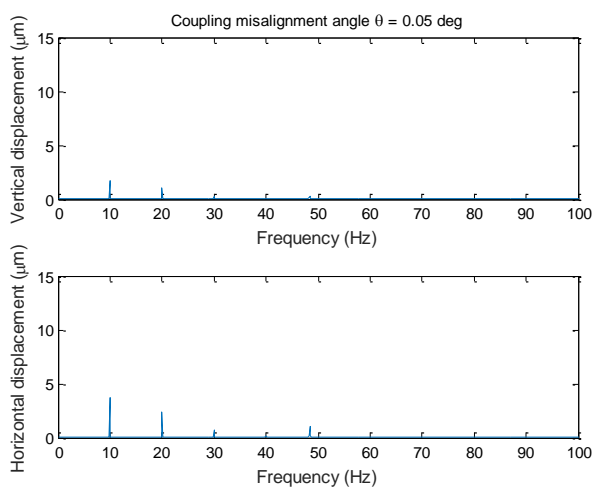

a)
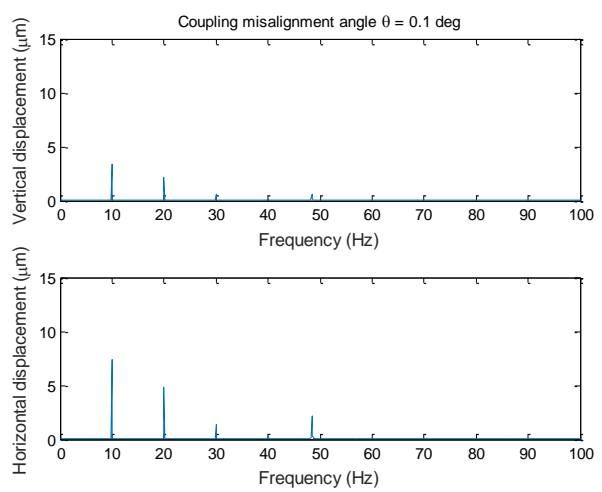

b)
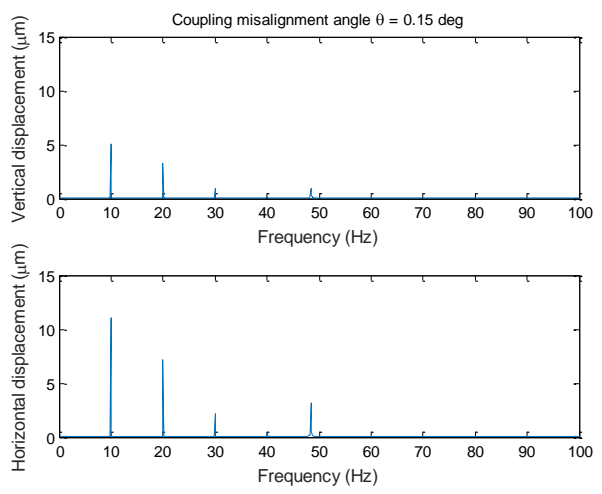

c)
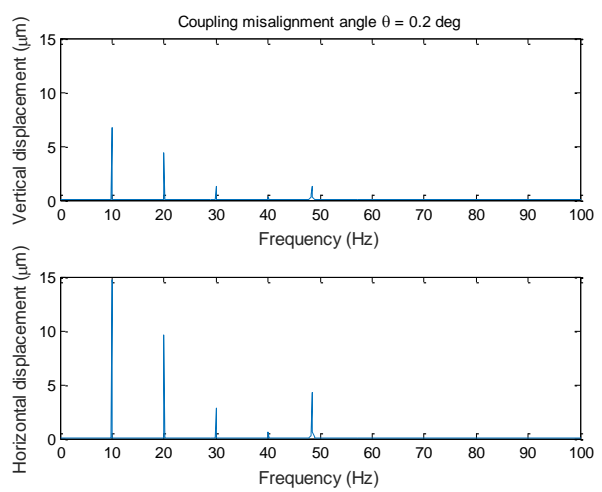

d)

Figure 6. Frequency spectrum of disk vibration as a function of coupling misalignment angle $(f=10 \mathrm{~Hz})$ 

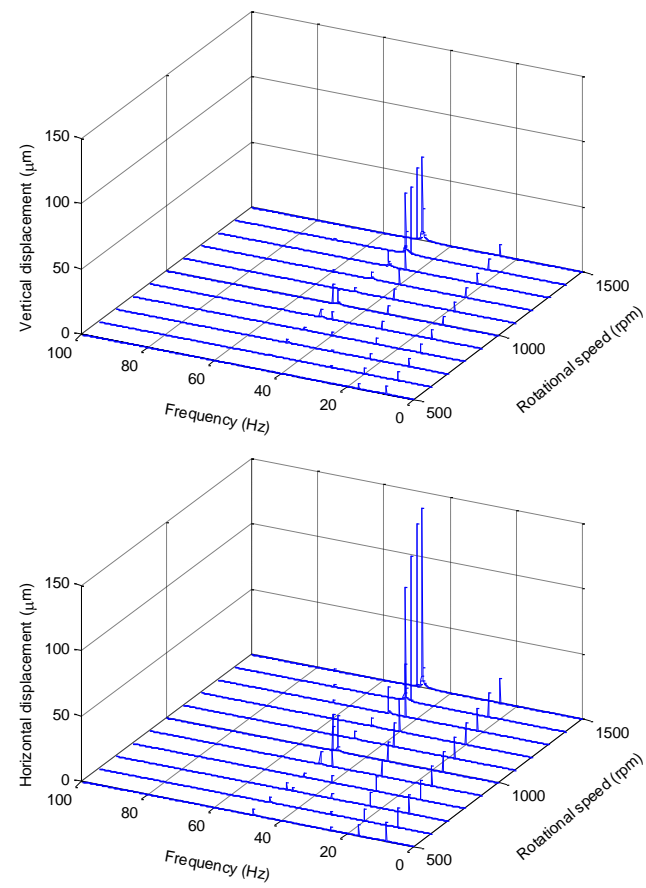

Figure 7. Frequency spectrum of disk vibration as a function of rotational speed $(\theta=0.2 \mathrm{deg})$

\section{Effect of rotational speed on rotor vibration}

In this section, numerical results on the effects of rotational speed are presented. The angular misalignment of coupling is selected by $0.2 \mathrm{deg}$. Figure 7 shows the dependence of vibration spectrum of the disk on rotational speed of the rotor. When any one of the multiple harmonics of rotational frequency is at or near the system natural frequency, the resonance phenomenon occurs along with large vibration amplitude. At the rotational speed of 1000 $\mathrm{rpm}$, for example, the $3 \mathrm{X}$ frequency component is $50.01 \mathrm{~Hz}$, which is close to the system natural frequency. Therefore, a relatively large $3 \mathrm{X}$ vibration amplitude is observed. Similarly, large vibration occurs at $2 \mathrm{X}$ frequencies when rotor rotates at 1400 or $1500 \mathrm{rpm}$. In the current speed range of rotor, the $1 \mathrm{X}$ frequency range does not intersect with the natural frequency. Therefore, the rotational speed shows a minor effect on the $1 \mathrm{X}$ vibration amplitude. The $1 \mathrm{X}$ vibration amplitude slightly increases with increasing rotational speed due to the increase in the gyroscopic effect of the disk.

\section{Other effects on rotor vibration}

This section presents the effect of disk location, bearing distance and arrangement on the vibration response of the system. The bearing preload, rotational frequency and coupling misalignment angle are selected at $150 \mathrm{~N}, f=10$ $\mathrm{Hz}$, and $\theta=0.1 \mathrm{deg}$, respectively.

Figure 8 shows the effect of the disk position on its vibration amplitudes. The locations of bearings are fixed as shown in Fig. 1. The vibration amplitudes at the disk are small at the positions near the supporting bearings. Maximum amplitude of $3 \mathrm{X}$ component is obtained when the disk is located at the mid-span of the bearings. This result is slightly different from those of $1 \mathrm{X}$ and $2 \mathrm{X}$ components.
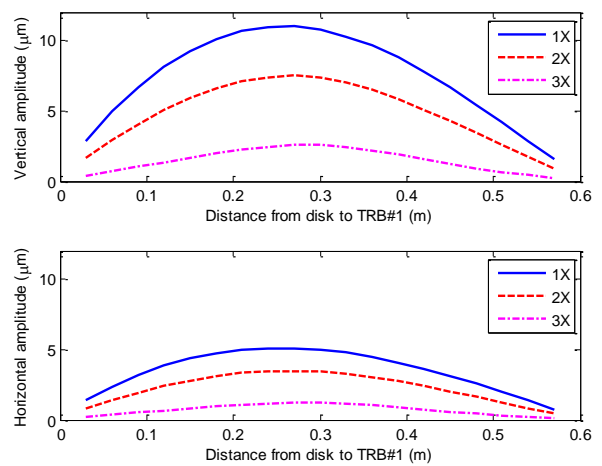

Figure 8. Effect of disk position on vibration amplitudes at the disk
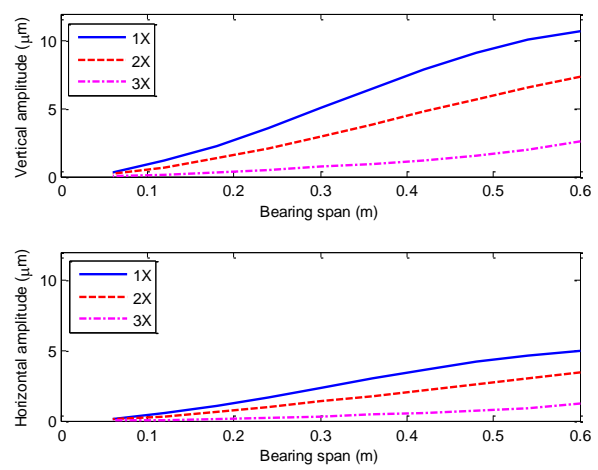

Figure 9. Effect of bearing distance on vibration amplitudes at the disk

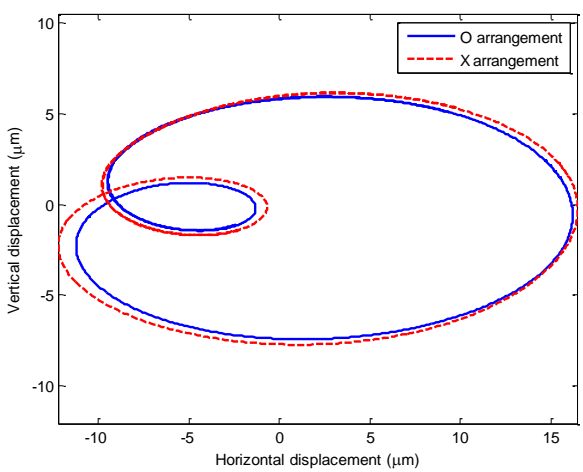

Figure 10. Effect of bearing arrangement on vibration orbit at the disk

Figure 9 shows the effect of bearing span distance on vibration amplitudes of the disk, which is fixed at the midspan of bearings. It is seen that the vibration amplitudes of all frequency components consistently increase with increasing the distance between two bearings. In general, the vibration of elements closed to bearing are small because of relatively high bearing stiffness.

Figure 10 illustrates the orbit of the disk center with two types of supporting bearing arrangements: face-to-face (or "X") arrangement and back-to-back (or "O") arrangement. This figure shows that the occurrence of angular misalignment in the coupling results in an orbit with looped pattern. The orbit shapes are almost identical for both arrangements. However, the "O" arrangement shows a smaller orbit size compared to that of the " $\mathrm{X}$ " arrangement. The reason is explained by the change in the bearing effective load center depending on the arrangement. 


\section{v. Conclusions}

A numerical model for calculation of coupling angular misalignment on vibration of rotor bearing system was developed and verified with the experimental measurement. Numerical simulations were performed to investigate the effects of coupling misalignment, rotational speed, disk position, bearings distance, and arrangement on the vibration response of the rotor. The angular misalignment in the flexible coupling introduces multiple harmonics of rotational frequency, which is attributed mainly by the periodic reaction forces at the coupling. The resonance conditions may occur whenever any one of the multiple harmonics is near the system natural frequency. The current analysis adopted constant bearing stiffness model. Further investigations can be made as a future work with considering the effect of varying bearing stiffness on vibration behavior of the system.

\section{Acknowledgment}

This research was supported by the Ministry of Trade, Industry \& Energy (MOTIE), Korea Institute for Advance of Technology (KIAT) through the Encouragement Program for the Industries of Economic Cooperation Region.

\section{References}

[1] P.N. Saavedra, and D. E. Ramirez, "Vibration analysis of rotors for the identification of shaft misalignment - part I: theoretical analysis," Proc. IMechE, C: J. Mech. Eng. Sci., vol. 218, pp. 971-985, 2004.

[2] T. H. Patel, and A. K. Darpe, "Vibration response of misaligned rotors," J. Sound Vib., vol. 325, pp. 609-628, 2009.

[3] T. H. Patel, M. J. Zuo, and A. K. Darpe, "Vibration response of coupled rotor systems with crack and misalignment," Proc. IMechE, C: J. Mech. Eng. Sci., vol. 225, pp. 700-713, 2010.

[4] AS Sekhar, and B.S. Prabhu, "Effects of coupling misalignment on vibrations of rotating machinery," J. Sound Vib., vol. 185, pp. 655671, 1995.

[5] S. Prabhakar, A. S. Sekhar, and A. R. Mohanty, "Crack versus coupling misalignment in a transient rotor system," J. Sound Vib., vol. 256, pp. 773-789, 2002.

[6] S. W. Hong, and V. C. Tong, "Rolling-element bearing modeling: a review," Int. J. Precis. Eng. Manuf., Vol. 17, pp. 1729-1749, 2016.

[7] Y. G. Jei, and C. W. Lee, "Modal analysis of continuous asymmetrical rotor-bearing systems," J. Sound Vib., vol. 152, pp. 245-262, 1992.

[8] V. C. Tong, and S. W. Hong, "Characteristics of tapered roller bearing with geometric error,” Int. J. Precis. Eng. Manuf., Vol. 16, pp. 27092716, 2015.

[9] V. C. Tong, and S. W. Hong, "Characteristics of tapered roller bearing subjected to combined radial and moment loads," Int. J. Precis. Eng. Manuf.-Green Tech., Vol. 1, pp. 323-328, 2014. 